\title{
Avaliação da coordenação e destreza motora - ACOORDEM: etapas de criação e perspectivas de validação*
}

\section{The assessment of motor coordination and dexterity: test development and validation perspectives}

\author{
Lívia C. Magalhães ${ }^{1}$, Viviane C.S. Nascimento², Márcia B. Rezende ${ }^{3}$
}

\begin{abstract}
MAGALHÃES, L. C.; NASCIMENTO, V. C. S.; REZENDE, M. B. Avaliação da coordenação e destreza motora - ACOORDEM: etapas de criação e perspectivas de validação. Rev. Ter. Ocup. Univ. São Paulo, v. 15, n. 1, p. 17-25, jan./abr., 2004.

RESUMO: Problemas de coordenação motora são comuns na infância e podem influenciar o desempenho escolar e a auto-estima da criança. Existem vários testes para deteç̧ão de problemas motores, mas nenhum deles foi criado e estandardizado para a criança brasileira. Na ausência de instrumentação apropriada para diagnóstico e caracterização das dificuldades motoras, muitas crianças não recebem ajuda, aumentando o risco de fracasso escolar. Nesse trabalho descrevemos as etapas iniciais de criação da Avaliação da Coordenação e Destreza Motora (ACOORDEM), teste para detecção de transtorno da coordenação motora em crianças de 4 a 8 anos de idade. A metodologia incluiu a revisão dos principais testes utilizados para avaliação da coordenação motora em crianças e criação de banco de dados com 349 itens de teste. Itens similares foram descartados e os restantes submetidos a critérios de inclusão, baseados nos princípios teóricos que nortearam a criação da ACOORDEM. Criou-se a versão inicial do teste, cuja validade de conteúdo foi avaliada por pesquisadores e profissionais que atuam na área de desenvolvimento infantil.
\end{abstract}

DESCRITORES: Destreza motora. Transtornos das habilidades motoras/terapia. Desenvolvimento infantil/métodos. Terapia ocupacional/tendências. Validade. Estudos de avaliação.

\footnotetext{
* Partes deste trabalho foram apresentadas como tema livre no V Encontro Mineiro de Avaliação Psicológica (Belo Horizonte, 2 a 5 de outubro de 2001) e no VIII Congresso Brasileiro de Terapia Ocupacional (Foz do Iguaçu, 2 a 6 de junho, 2003).

${ }^{1}$ Doutora em Educação pela Universidade de Illinois - EUA, Docente do Curso de Terapia Ocupacional /Universidade Federal de Minas Gerais.

${ }^{2}$ Terapeuta ocupacional, formada pela Universidade Federal de Minas Gerais.

${ }^{3}$ Doutora em Fisiologia pela Universidade Federal de Minas Gerais, Docente do Curso de Terapia Ocupacional/Universidade Federal de Minas Gerais.

Endereço para correspondência: Departamento de Terapia Ocupacional. Avenida Antônio Carlos, $\mathrm{n}^{\circ} 6627$ - Unidade Administrativa II, $3^{\circ}$ andar - Campus Pampulha, Belo Horizonte, MG, CEP: 31270-901. e -mail: liviam@ gcsnet.com.br
} 
$\mathrm{T}$ rabalhando na área de terapia ocupacional infantil há muitos anos, nosso interesse em criar um teste de desenvolvimento motor parte da insatisfação com o tipo de instrumentação disponível no Brasil para a avaliação de distúrbios sutis da coordenação e destreza motora em crianças. Apesar de encontrarmos na literatura referências a uma variedade de testes motores (AYRES, 1972, 1980; BRUININKS, 1978; FOLIO; FEWELL, 1983; HENDERSON; SUGDEN, 1992; MILLER, 1982, 1993), não existem testes padronizados de desenvolvimento motor global, com normas para crianças brasileiras em idade escolar, e muito menos instrumentos específicos para detecção de transtornos da coordenação. Portanto, temos acesso a uma variedade de técnicas de intervenção motora, tais como a terapia de Integração Sensorial (AYRES, 1972; BUNDY et al., 2002), o treino perceptual motor (KEPARTH, 1986), as estratégias sensório-motoras para melhoria da função manual (BENBOW, 1995; MEYERS, 1992) ou o treinamento motor cognitivo (MARTINI; POLATAJKO, 1998), mas um problema persiste: como avaliar se a criança de fato tem um transtorno da coordenação motora e como medir os resultados da intervenção?

Diante desta situação, qual seria a melhor solução: criar um teste novo ou usar os já existentes? Apesar de conveniente, o uso de testes importados, além do alto custo, tem limitações, pois a maioria apresenta problemas de validade quando aplicados em populações diferentes daquelas para as quais foram criados (CROW et al., 1999). Uma solução seria validar para a criança brasileira instrumentos já consagrados, como o Movimento ABC (HENDERSON; SUGDEN, 1992) ou o teste Bruininks Ozeretski de Proficiência Motora (BRUININKS, 1978). O problema, no entanto, seria o custo desse processo e dos kits de testes, que são caros para o nosso padrão econômico. Além disso, os editores de testes geralmente cobram taxas consideráveis para o direito de tradução.

Face à necessidade de melhorar os recursos disponíveis para a avaliação do desenvolvimento motor de crianças brasileiras e considerando as dificuldades relacionadas ao uso de testes importados, decidimos criar um teste de desenvolvimento motor e validá-lo em nosso país. O objetivo desse estudo, portanto, é descrever o processo de criação da Avaliação da Coordenação e Destreza Motora - ACOORDEM
(MAGALHÃES, REZENDE, 2001), um teste específico para detecção de problemas de coordenação motora em crianças de 4 a 8 anos de idade.

\section{O transtorno do desenvolvimento da coorde- nação motora: definição e caracterização}

Estudados desde o início do século passado e descritos sob vários nomes, tais como disfunção cerebral mínima (DCM), hipercinesia, distúrbio perceptual motor, síndrome da criança desajeitada, agnosia e apraxia do desenvolvimento, dispraxia do desenvolvimento, somatodispraxia e distúrbio psicomotor (AYRES, 1972; GUBBAY, 1975; GARCÍA, 1998; KEPARTH, 1986; MARTINI; POLATAJKO, 1998), os problemas de coordenação motora na criança recentemente receberam um diagnóstico formal: Transtorno do Desenvolvimento da Coordenação (Developmental Coordination Disorder DCD) ou TDC, para abreviar. O termo TDC apareceu pela primeira vez no Manual Estatístico de Diagnóstico de Distúrbios Mentais DSM IIII-R em 1987 e, atualmente, tem a classificação 315.4 no DSM IV ${ }^{(1)}$, constando no CID-10(2), com a classificação F82. O diagnóstico de TDC se aplica aos casos em que, na ausência de outros distúrbios físico/neurológicos conhecidos, existe um prejuízo acentuado do desenvolvimento da coordenação motora, com desempenho significativamente abaixo do esperado para a idade cronológica e nível cognitivo da criança, nas atividades diárias que requerem coordenação motora (DAVID, 1995; MARTINI; POLATAJKO, 1998).

Os sinais geralmente associados à TDC são: desajeitamento e inconsistência no desempenho de tarefas, coordenação motora pobre, problemas de ritmo e na transferência de aprendizagem, declínio do desempenho com a repetição, tensão corporal e excesso de atividade muscular na execução de tarefas motoras (SELLER, 1995). A criança pode demonstrar dificuldade nas tarefas de auto-cuidado, como vestir e usar utensílios, nas atividades acadêmicas, como a escrita e o traçado, e nas atividades de lazer, com pobre desempenho em esportes, no uso de equipamentos de parques e brinquedos infantis, além de problema nas interações sociais (MARTINI; POLATAJKO, 1998). Apesar de não existir estatística brasileira, estima-se que entre 5 a $10 \%$ das crianças em idade escolar apresentem TCD (CERMAK et al., 2002; DAVID, 1995; MARTINI; POLATAJKO, 1998),

\footnotetext{
(1) Manual de diagnóstico e estatística de distúrbios mentais (DSM-IV). Porto Alegre: Artes Médicas, 1994.

(2) Organização Mundial da Saúde. CID-10. $8^{a}$.ed. 10 rev. São Paulo: Edusp, 2000. 3v.
} 
sendo, portanto, uma condição bastante freqüente no ambiente escolar.

A literatura sobre o TDC é vasta, sendo evidente que essa é uma condição que persiste até a idade adulta, tendo impacto tanto na vida escolar e social da criança, como nos outros membros da família (LOSSE et al., 1991; MARTINI; POLATAJKO, 1998; STEPHENSON et al., 1991; MISSIUMA; POLATAJKO, 1995). Apesar de não haver consenso sobre as causas da TDC, uma área já bastante pesquisada, é a relação entre prematuridade/baixo peso e os distúrbios da coordenação (CHERKES-JULKOWSKI, 1998; GOYEN et al., 1998; LUOMA et al., 1998; MAGALHÃES et al., 1999; MAGALHÃES et al., 2003). Independente da causa, a prevenção e detecção do TDC exige avaliação criteriosa, com uso de testes específicos para diagnóstico desse tipo de problema.

Henderson (1987) identifica três abordagens tradicionalmente usadas na avaliação dos distúrbios da coordenação em crianças: os testes descritivos, os testes diagnósticos e os testes de desenvolvimento neurológico. Testes descritivos, também denominados orientados para produto, são aqueles caracterizados por listas de habilidades organizadas de maneira cronológica. Em geral são instrumentos neutros, sob o ponto de vista teórico, podendo ser usados por vários profissionais, e os itens do teste avaliam se a criança é capaz ou não de fazer certas tarefas funcionais, como agarrar uma bola e cortar com a tesoura (HENDERSON, 1987). A maioria dos testes de desenvolvimento se enquadra nessa categoria, como por exemplo, o Teste de Desenvolvimento Denver II (FRANKENBURG et al., 1994) e as Escalas Bayley de Desenvolvimento Infantil (BAYLEY, 1993). Na área específica de avaliação da criança escolar, destacam-se o Bruininks Ozeretsky Teste de Proficiência Motora - BOTMP (BRUININKS, 1978) - e o Peabody (FOLIO; FEWELL, 1983). Um instrumento recente, lançado no Brasil, é a avaliação motora da criança (ROSA NETO, 2002), que apesar de reportar normas por idade, não pode ser considerada uma avaliação brasileira, pois os dados foram coletados fora do país.

Os testes diagnósticos, em sua maioria, foram criados por educadores ou terapeutas, e refletem algum tipo de orientação teórica, sendo voltados para a avaliação dos componentes perceptuais e sensoriais, considerados substratos da atividade motora (HENDERSON, 1987). Nessa categoria se enquadram testes perceptual-motores, tais como o Bender (KOPPITZ, 1989), o Teste de Integração Visomotora (BEERY, 1997) e o teste perceptual-motor de Keparth
(ROACH; KEPARTH, 1966), que são bastante conhecidos no Brasil, dentro da abordagem perceptual motora de tratamento dos distúrbios de aprendizagem. Ainda nessa categoria, se enquadram os testes de integração sensorial desenvolvidos por terapeutas ocupacionais (AYRES, 1980, 1989; MILLER, 1982, 1993), mas nenhum deles foi traduzido para o português.

$\mathrm{Na}$ categoria de testes de desenvolvimento neurológico, estão os instrumentos criados por médicos, na sua maioria adaptações do exame neurológico evolutivo, sem maiores preocupações com estudos de estandardização ou normatização (HENDERSON, 1987). Além dos instrumentos importados, como o Exame Neurológico Rápido Quick/QNST (MUTTI et al., 1978) -, existem alguns exames brasileiros, como o Exame Neurológico Evolutivo (LEFÈVRE, 1976) ou, em uma versão recente, a Avaliação Neurológica Infantil (COELHO, 1999).

$\mathrm{O}$ teste que pretendemos criar se enquadra na categoria de teste descritivo, pois pretendemos documentar o desenvolvimento motor da criança de 4 a 8 anos de idade. Nossa intenção ao criar um teste descritivo, é que ele possa ser usado, inteiro ou por partes, pelos vários profissionais que têm interesse em documentar o desenvolvimento da coordenação motora em crianças nessa faixa etária. Naturalmente nossa formação e fundamentação em teorias específicas da área de terapia estarão moldando o instrumento, mas não estamos, nesse momento, nos apegando a correntes ou métodos específicos de tratamento, de forma que o teste possa ter uma utilização mais ampla.

\section{METODOLOGIA}

Para a criação da ACOORDEM adotamos a metodologia proposta por Benson e Clark (1982), para desenvolvimento de testes na área de terapia ocupacional. Segundo esses autores o processo de desenvolvimento de testes inclui quatro etapas:

(a) Fase I - Planejamento: quando é definido o objetivo e tipo de população alvo do teste, sendo feita uma ampla revisão da literatura sobre instrumentos disponíveis no mercado, tipos de itens e formato de teste;

(b) Fase II - Construção: os itens são desenvolvidos, criando um banco de dados, que devem, então, ter a validade de conteúdo avaliada por um painel de profissionais/ pesquisadores experientes na área (Painel de experts). Os itens são então 
selecionados e revisados, novos itens podem ser incluídos, sendo feita a primeira versão piloto do teste;

(c) Fase III - Avaliação quantitativa: a versão piloto passa por uma revisão geral, sendo então aplicada em um grupo experimental apropriado. São realizadas análises para examinar confiabilidade, cujos resultados serão usados para nova revisão do instrumento. É feita uma segunda aplicação piloto do teste, com exame da confiabilidade, nova revisão e assim sucessivamente até se chegar ao formato final;

(d) Fase IV - Validação: o instrumento é aplicado em um número significativo de crianças, sendo coletados dados para derivação de normas, exame da validade concorrente, de critério e de construto.

Nesse estudo descreveremos apenas as etapas I e II de criação da ACOORDEM, uma vez que o teste ainda está em desenvolvimento. Trabalhos futuros discutirão as etapas de avaliação quantitativa e validação.

\section{Fase I - Planejamento}

O trabalho foi iniciado, com extensa revisão da literatura, enfocando a caracterização dos transtornos da coordenação e os instrumentos usados para detecção e diagnóstico desses problemas na criança, como já discutido brevemente. Visando justificar a necessidade de se desenvolver um teste de coordenação motora infantil, fizemos também um levantamento informal da frequiência de problemas de coordenação em algumas escolas de ensino fundamental de Belo Horizonte (BH). Um total de 91 professores, de três escolas particulares e três escolas públicas, responderam ao questionário sobre a freqüência de crianças com dificuldade de coordenação entre os alunos da turma sob sua responsabilidade. Os resultados indicaram freqüência variando de 3 a 19\% de crianças com algum tipo de dificuldade de coordenação, em turmas do primeiro período da educação infantil ao $4^{\circ}$ ano do ensino fundamental. Metade das professoras $(52,7 \%)$ indicaram que os problemas de coordenação motora são freqüentes, sendo comum encontrarem duas ou mais crianças com dificuldade em cada turma. Esse levantamento de dados nas escolas foi apenas um estudo preliminar, realizado sem grande rigor metodológico, mas nos deu uma visão sobre os problemas de coordenação motora na sala de aula e nos incentivou a dar continuidade ao processo de criação do teste. Estudo mais detalhado, incluindo amostragem representativa de escolas públicas da Grande $\mathrm{BH}$, está em andamento.

Confirmada a necessidade de criação do teste, passamos à definição dos objetivos e características do instrumento. Nosso objetivo era criar um teste para detecção de problemas de coordenação motora em crianças de 4 a 8 anos. Esse instrumento deveria ser de fácil aplicação, podendo ser usado por profissional recém-formado a partir do manual de instruções. O teste deveria ser compatível com os objetivos e princípios de atuação do terapeuta ocupacional com crianças e deveria contemplar a avaliação de habilidades nas três áreas de função, como definido pela Classificação Internacional de Funcionalidade (WORLD HEALTHORGANIZATION, 2001):

- estrutura e função do corpo: incluindo itens puramente motores, tais como, equilíbrio, força, coordenação motora e padrões de preensão;

- atividades: incluindo a observação direta ou indireta (questionário) da criança desempenhando atividades típicas escolares, tais como, escrita, recorte, traçado;

- participação: observação indireta, através de questionários para pais e professores, do desempenho da criança nas atividades escolares, rotinas de vida diária em casa e na escola, além dos interesses e preferências no brincar.

Definidos os objetivos do instrumento, foi feita a revisão dos principais testes de coordenação motora, usados na área infantil. Demos preferência a testes padronizados, dado que nosso objetivo é desenvolver teste normativo. Boa parte dos testes revisados estavam disponíveis na Biblioteca de Testes do Laboratório de Atividade e Desenvolvimento Infantil (LADIN), do Departamento de Terapia Ocupacional da UFMG. Passamos então para a Fase II, de construção do teste.

\section{Fase II - Construção do teste}

Como indicado na Tabela 1, analisamos 13 testes motores, cujos itens foram traduzidos, compondo um banco com 349 itens. Itens de protocolos de avaliação brasileiros, como o Exame Neurológico Evolutivo (LEFÈVRE, 1976) e a Avaliação Neurológica Infantil (COELHO, 1999) foram examinados, mas como já estavam contemplados nos itens dos testes padronizados, eles não foram contabilizados na Tabela 1 . 
Tabela 1 - Testes revisados para compor o banco de itens da ACOORDEM.

\begin{tabular}{|c|c|}
\hline Testes revisados & Número de itens* \\
\hline 1. $\quad$ Bruininks-Oseretsky Test of Motor Proficiency ${ }^{9}$ & 48 \\
\hline 2. $\quad$ First Step ${ }^{37}$ & 12 \\
\hline 3. $\quad$ Miller Assessment for Preschoolers ${ }^{36}$ & 27 \\
\hline 4. Movimento ABC (Movement Assessment Battery For Children) ${ }^{22}$ & 32 \\
\hline 5. Peabody Developmental Motor Scale ${ }^{16}$ & 74 \\
\hline 6. Pediatric Early Elementary Examination - PEEX ${ }^{26}$ & 32 \\
\hline 7. Pediatric Examination of Educational Readiness - PEER ${ }^{27}$ & 29 \\
\hline 8. Quick Neurological Screening Test ${ }^{39}$ & 15 \\
\hline 9. Sensory Integration and Praxis Tests - SIPT $^{3}$ & 16 \\
\hline 10. Southern California Sensory Integration Tests - SCSIT $^{2}$ & 14 \\
\hline 11. Teste de Gubbay de Proficiência Motora ${ }^{20}$ & 7 \\
\hline 12. The Purdue Perceptual-Motor Survey ${ }^{40}$ & 19 \\
\hline 13. Visual Motor Integration Test $-\mathrm{VMI}^{5}$ & 24 \\
\hline TOTAL & 349 \\
\hline
\end{tabular}

Nota: * Número de itens se refere à categoria do item, isto é, motor, perceptual, praxia, e não ao número de sub-itens que compõem cada item dos testes.

Em uma segunda etapa, os 349 itens catalogados foram agrupados em categorias, definidas de acordo com a classificação do item em cada teste. Na Tabela 2 apresentamos as categorias nas quais os itens foram agrupados. Observamos que havia muitos itens repetidos, havendo um grande número de itens similares nas áreas de equilíbrio, provas com bola e salto. É interessante observar que, apesar da maioria dos testes analisados serem voltados para avaliação das habilidades motoras, encontramos um grande número de itens de percepção visual, incluindo coordenação visomotora, além de vários itens cognitivos.

Tabela 2 - Número de itens obtidos por área catalogada.

\begin{tabular}{lc}
\hline Classificação dos itens por área & Número de itens \\
\hline Provas Neurológicas & \\
\hline Tônus Postural & 11 \\
- Movimentação das Extremidades & 12 \\
Força & 10 \\
Velocidade Tempo de Reação & 4 \\
Equilíbrio & 42 \\
Salto & 27 \\
Sequenciamento motor - Corporal & 13 \\
Sequenciamento motor - Manual & 5 \\
Provas com Bola & 32 \\
Destreza Manual & 27 \\
Recorte & 4 \\
Praxia & 13 \\
Discriminação Sensorial & 16 \\
Percepção Visual & \\
Coordenação Visomotora & 64 \\
Construção com Blocos & 6 \\
Percepção Visoespacial & 17 \\
Cognição & 44 \\
Outros & 2 \\
\hline Total de itens & $349 *$ \\
\hline
\end{tabular}

Feita a catalogação e classificação, contávamos com um número excessivo de itens, sendo necessário elaborar critérios para selecionar os itens de interesse para a criação do novo teste. Considerando que o objetivo era criar um teste para avaliar habilidades motoras, foram desenvolvidos critérios específicos de inclusão e exclusão de itens para compor a versão piloto da ACOORDEM. Os critérios usados para exclusão de itens foram:

- Duplicados ou repetidos em variações similares;

- tipicamente neurológicos;

- puramente visuais - existem testes específicos nessa área, sendo incluídos apenas os itens visomotores;

- puramente cognitivos ou de conteúdo eminentemente pedagógico;

- de difícil implementação, que exijam muito treinamento, com escore difícil ou que exijam muita experiência do examinador;

- que exigiam materiais difíceis, complicados ou caros;

- que necessitavam de equipamentos difíceis de serem transportados e usados em diferentes condições;

- sem nenhuma relevância cultural.

Os critérios para inclusão de itens no teste foram:

- Componente motor evidente;

- divertido para a criança;

- fácil de aplicar;

- fornece boas informações clínicas;

- forma de administração e escore confiáveis;

- a literatura dá suporte para inclusão no teste.

Aplicando esses critérios, selecionamos itens 
para compor, como que, um esqueleto do teste. Os itens selecionados foram revisados e agrupados em sub-áreas (i.e: coordenação bilateral, destreza manual, coordenação visomotora, etc.). Os itens da ACOORDEM não foram simplesmente copiados dos testes originais, em sua maioria foram adaptados, considerando a cultura, as atividades, os recursos e materiais de fácil acesso em nosso país. A versão piloto da ACOORDEM, com definição de áreas de teste, itens e sugestão de formato de escore, foi então submetida à avaliação externa da validade de conteúdo.

Foram organizados dois grupos de discussão, para apreciação dos itens do teste. O primeiro grupo (Painel de Experts), foi constituído por três pesquisadoras doutoras (duas neuropediatras e uma terapeuta ocupacional) e dois profissionais (uma terapeuta ocupacional e uma educadora), com larga experiência na área de avaliação infantil, além da equipe de pesquisa da ACOORDEM. Esse grupo avaliou aspectos técnicos do teste. O segundo grupo de discussão (Painel Clínico), foi constituído por oito terapeutas ocupacionais, com grande experiência no tratamento de crianças que apresentam transtorno da coordenação motora. Esse grupo avaliou aspectos práticos do teste, dando sugestões sobre a facilidade de aplicação dos itens, sua utilidade clínica, além de sugerir materiais alternativos para composição do kit de teste. A ACOORDEM foi bem avaliada nos dois painéis, sendo feitas apenas sugestões práticas, visando facilitar a aplicação e melhorar a utilidade clínica do teste. Nenhum item foi acrescentado ou excluído, mas os critérios de escore e forma de aplicação de alguns itens foram modificados.

\section{RESULTADO}

O resultado do trabalho desenvolvido nas fases I e II de criação da ACOORDEM foi a primeira versão do teste. As recomendações dos painéis foram incorporadas ao instrumento, que foi novamente revisado, originando o teste, dividido em três áreas:

a) Coordenação e destreza manual - Observação direta (23 itens)

- Movimentação fina dos dedos - Brincando com as mãos - itens que avaliam a velocidade e destreza manual, com ênfase nos movimentos de manipulação na mão;

- acuidade do traçado - Brincando de desenhar provas cronometradas de traçado em circuitos;

- manejo da tesoura-Brincando de recortar-provas cronometradas de recorte simples e complexo;

- sensação tátil - Brincando de adivinhar - provas de discriminação de objetos e de dedos;

- escrita - Brincando de escrever - prova cronometrada de escrita do alfabeto.

b) Coordenação corporal e planejamento motor Observação direta (26 itens)

- Coordenação bilateral - Brincando com bola brincadeiras de agarrar, rebater e arremessar bola;

- equilíbrio e postura - Brincando de equilibrar circuitos dinâmicos, exigindo várias habilidades de equilíbrio;

- sequenciamento - Brincando de pular - provas de sequenciamento motor, incluindo atividades similares à amarelinha e brincadeira de batucada;

- planejamento motor - Brincando de macaco disse provas de imitação de posturas e representação de ações.

c) Desempenho funcional em casa e na escola-Através de questionários estruturados

Questionário para Professores - 68 itens, com escore em 4 pontos.

- Habilidade para expressar o que está aprendendo;

- habilidade para assumir o papel de estudante;

- habilidades de auto-cuidado;

- mobilidade e estabilidade postural.

Questionário para Pais - 56 itens, com escore em 4 pontos.

- Habilidade para brincar e preferências lúdicas;

- desempenho das atividades de vida diária;

- estabelecimento de hábitos e rotinas.

Os itens de observação direta (áreas de Coordenação e Destreza Manual e de Coordenação Corporal e Planejamento Motor) têm escore numérico, baseado no tempo e acuidade da resposta. Os questionários de pais e professores (Desempenho funcional em casa e na escola) serão pontuados em escala de quatro pontos, indicando a freqüência dos comportamentos observados (nunca/raramente, ocasionalmente, freqüentemente e sempre). Como indicado pelos nomes dos itens, procuramos dar ao teste um formato de brincadeira, visando facilitar sua aceitação pelas crianças. A ACOORDEM versão 1.0 é extensa, mas no processo de validação do instrumento, muitos itens serão descartados, devendo ser mantidos apenas aqueles de melhor qualidade psicométrica. Concluídas as fases I e II do processo de criação do teste, a ACOORDEM 1.0 está pronta para darmos início aos estudos para examinar a confiabilidade e validade dos itens.

futuros

\section{Conclusão e rocomendações para estudos}

A criação de testes válidos e confiáveis de 
aspectos do desempenho humano é um processo longo. Para chegarmos à ACOORDEM 1.0 trabalhamos cerca de dois anos, mas temos um caminho ainda mais longo pela frente, de lapidação e validação do teste até o produto final, pronto para ser usado na clínica. Na próxima etapa, de avaliação quantitativa, o teste deverá ser pilotado em pequenas amostragens, para análise de itens, com exame da confiabilidade (entre observadores e teste-reteste) e aspectos iniciais da validade de construto. Nesse processo pretendemos usar a metodologia Rasch (BOND, 2001; WRIGHT, 1979), específica para criação de testes e medidas. Como resultado da fase III, esperamos eliminar e ajustar alguns itens, para entrarmos na fase final, de validação, com um teste mais enxuto, de fácil aplicação pelos terapeutas.

Apesar da ACOORDEM 1.0 ter recebido comentários positivos nos dois painéis de avaliação da validade de conteúdo, um aspecto ainda merece consideração. Como um teste formal, a ACOORDEM é voltada para a avaliação do desempenho da criança por terceiros, seja pelo examinador, através dos itens de observação direta, seja pelos pais e professores, através de questionários, não havendo ainda espaço para expressão individual da criança. É nossa intenção, no processo de desenvolvimento do teste, criar estratégia que permita "dar voz à criança", para que ela expresse sua perspectiva sobre o problema avaliado. Ainda não temos uma boa solução para essa questão, mas estamos examinando outros testes.

Enfim, temos um trabalho longo pela frente e possivelmente desenvolveremos a ACOORDEM em etapas, trabalhando com áreas individuais (Destreza Manual, Coordenação Corporal e Desempenho em Casa e na Escola), avançando inicialmente na área de Destreza Manual, para a qual há menos instrumentos padronizados disponíveis e sentimos necessidade de avaliação mais precisa. Como ainda não existem testes normativos para crianças brasileiras nas áreas de desempenho examinadas pela ACOORDEM, esperamos que nosso trabalho venha a contribuir para a detecção e melhoria da atenção às crianças que apresentam transtorno da coordenação motora.

Esse é um projeto a longo prazo, que vai exigir o trabalho de várias pessoas. O ideal seria desenvolver o projeto em colaboração com pesquisadores de outros estados, o que permitiria a coleta de dados em regiões mais amplas do país. Nosso maior desafio, no entanto, é localizar fontes de financiamento que permitam dar continuidade ao projeto, ou, de maneira mais ambiciosa, que dêem suporte para trabalhos em parceria, abrindo perspectiva para expandi-lo, com inclusão de dados de crianças de diferentes estados brasileiros.

MAGALHÃES, L. C.; NASCIMENTO, V. C. S.; REZENDE, M. B. The assessment of motor coordination and dexterity: test development and validation perspectives. Rev. Ter. Ocup. Univ. São Paulo, v. 15, n. 1, p. 17-25, jan./abr., 2004.

\begin{abstract}
Problems in motor coordination are common in infancy and they may influence the child's school performance and self-stem. There are various tests designed to detect motor problems, but none of them was created and standardized for the Brazilian children. In the absence of appropriate instruments to diagnose and characterize motor problems, the children do not get assistance, increasing the risk for school failure. In this study we describe the initial steps of the creation of the Assessment of Motor Coordination and Dexterity (ACOORDEM), a test designed to detect developmental coordination disorder in children ages 4 to 8 years old. The methodology included a review of the most well known tests that are used to assess motor coordination in children and the creation of a item bank, with 349 entries. Items that were similar were discarded and the remaining were submitted to inclusion criteria, based on theoretical principles that guided the creation of the ACOORDEM. An initial version of the test was created, and its content validity was examined by panels of experts and clinicians, involved in the area of child development.
\end{abstract}

KEY WORDS: Motor skills. Motor skills disorders. Child development/trends. Occupational therapy/trends. Occupational therapy/methods. Validity. Evaluation study.

Reconhecimento: Esse projeto foi financiado com recursos do Conselho Nacional de Desenvolvimento Científico e Tecnológico - CNPq e da Fundação de Amparo à Pesquisa de Minas Gerais - FAPEMIG. 


\section{REFERÊNCIAS}

1. AYRES, A. J. Sensory integration and learning disorders. Los Angeles: WPS, 1972.

2. AYRES, A. J. Southern California sensory integration tests revised. Los Angeles: WPS, 1980.

3. AYRES, A. J. Sensory integration and praxis tests. Los Angeles: Western Psychological Services, 1989.

4. BAYLEY N. Bayley scales of infant development. 2nd ed. San Antonio: The Psychological Corporation, 1993.

5. BEERY, K. E. Revised administration, scoring, and teaching manual for the developmental test of visualmotor integration. Parsippany, NJ: Modern Curriculum Press, 1997.

6. BENBOW, M. Principles and practices of teaching handwriting. In: HENDERSON, A; PEHOSKI, C. (Ed.). Hand function in the child: foundation for remediation. St. Louis, MO: Mosby, 1995. p.295-1.

7. BENSON, J.; CLARK, F. A guide to instrument development and validation. Am. J. Occup. Ther., v. 36, n. 12, p.789-800, 1982.

8. BOND, T. G.; FOX. C. M. Applying the Rasch model: fundamental measurement in the human sciences. Mahwah, N J: Laurence Earlbaum Associates, 2001.

9. BRUININKS, R. H. Bruininks-Oseretsky test of motor proficiency. Circle Pine: American Guidance Service, 1978.

10. BUNDY, A. C.; LANE, S. S. J.; MURRAY, E. A. Sensory integration: theory and practive. $2^{\mathrm{a}}$ ed. Philadelphia: $\mathrm{F}$ A. DAVIS, 2002.

11. CERMAK, S. A.; GUBBAY, S. S.; LARKIN, D. What is developmental coordination disorder?. In: CERMAK, S. A.; LARKIN, D. (Ed.). Developmental coordination disorder. Albany, NY: Delmar, 2002.

12. CHERKES-JULKOWSKI, M. Learning disability, attention deficit disorder, and language impairment as outcomes of prematurity: a longitudinal descriptive study. J. Learn. Disabil., v. 31, n. 3, p. 294-306, 1998.

13. CHOW, S. M. K.; HENDERSON, S. E.; BARNETT, A. L. The movement assessment battery for children: a comparison of 4-year-old to 6-year-old from Hong Kong and the United States. AJOT, v. 55, n. 1, p. 55-61, 2001.

14. COELHO, M. S. Avaliação neurológica infantil nas ações primárias de saúde. Rio de Janeiro: Atheneu, 1999.

15. DAVID, K.S. Developmental coordination disorder. In: CAMPBELL, S. Physical therapy for children. Philadelphia, WB Saunders, 1995.

16. FOLIO, M. R.; FEWELL, R. Peabody developmental motor scales. Allen, TX: DLM Teaching Resources, 1983.

17. FRANKENBURG, W. K.; DODDS, J.; ARCHER, P.; BRESNICK, B.; MASCHKA, P.; EDELMAN, N.; SHAPIRO, H. Denver II screening manual. Denver, CO: Denver Developmental Material, Inc., 1994.
18. GARCíA， J. N. Manual de dificuldades de aprendizagem. Porto Alegre: Artes Médicas, 1998.

19. GOYEN, T. A.; LUI, K.; WOODS, T. Visual-motor, visual-perceptual, and fine motor outcomes in very-lowbirthweight children at 5 years. Dev. Med. Child Neurol. v. 40, p.70-81, 1998.

20. GUBBAY, S. S. The clumsy child: a study of developmental apraxic and agnosic ataxia. London: WB Saunders, 1975.

21. HENDERSON, S. E. The assessment of the "clumsy" children: old and new approaches. J. Child Psychol. Psychiatry, v. 28, n. 4, p.511-27, 1987.

22. HENDERSON, S.E.; SUGDEN, D.A. Movement assessment battery for children. Londres: The Psychological Corporation, 1992.

23. KEPARTH, N. O aluno de aprendizagem lenta. Porto Alegre: Artes Médicas, 1986.

24. KOPPITZ E. O teste gestáltico de Bender para crianças. $2^{\mathrm{a}}$ ed. Porto Alegre: Artes Médicas, 1989.

25. LEFÈVRE, A. B. Exame neurológico evolutivo. São Paulo: Sarvier, 1976.

26. LEVINE, M. D; RAPPAPORT, L. Pediatric early elementary examination - PEEX. Cambridge: EPS, 1983.

27. LEVIne, M. D.; SCHNEIDER, E. A. Pediatric examination of educational readiness - PEER. Cambridge: EPS, 1983.

28. LOSSE, A.; HENDERSON, S. E.; ELLIMAN, D.; HALL, D.; KNIGHTS, E.; JONGMANS, M. Clumsiness in children: do they grow out of it? Dev. Med. Child Neurol., v. 33, p. 55-68, 1991.

29. LUOMA, L.; HERRGARD, E.; MARTIKAINEN, A. Neuropsychological analysis of the visuomotor problems in children born preterm at $<32$ weeks of gestation: a 5year prospective follow-up. Dev. Med. Child Neurol., v. 40, p. 21-30, 1998.

30. MAGALHÃES, L. C.; BARBOSA, V. M.; LOPES, K. C.; PAIXÃO, M. L. Estudo longitudinal do desenvolvimento de recém-nascidos pré-termo: avaliação na idade pré-escolar. Rev. Bras. Neurol., v. 35, n. 4, p. 87-93, 1999.

31. MAGALHÃES, L. C.; REZENDE, M. B. Avaliação da coordenação e destreza motora - ACOORDEM - Versão 1. Manuscrito não publicado. Belo Horizonte: Departamento de Terapia Ocupacional, UFMG, 2001.

32. MAGALHÃES, L. C.; WENDLING, P. C.; PAIXÃO, M. L.; MANCINI, M. C.; BARBOSA, V. M. Estudo comparativo sobre o desempenho perceptual e motor na idade escolar em crianças nascidas pré-termo e a termo. Arq. Neuropsiquiatr. v. 61, p. 250-5, 2003.

33. MANCINI, M. C.; PAIXÃO, M. L.; SILVA, T. T; MAGALHÃES, L. C.; BARBOSA, V. M. Comparação 
das habilidades motoras de crianças prematuras e crianças nascidas a termo. Rev. Fisioter. Univ. São Paulo, v.7, p. 25-31, 2000.

34. MARTINI, R. H.; POLATAJKO, H. J. Verbal selfguidance as a treatment approach for children with developmental coordination disorder: a systematic replication study. Occup. Ther. J. Res., v. 18, n. 4, p. 157-81, 1998.

35. MEYERS, C. A. Therapeutic fine-motor activities for preschoolers. In: CASE-SMITH, J.; PEHOSKI, C. Development of hand skills in the child. Rockville, MD: AOTA, 1992.

36. MILLER, L. J. Miller assessment for preschoolers. Little Town, CO: Foundation for Knowledge in Development, 1982.

37. MILLER, L. J. FirstSTEP: screening test for evaluating prescholers. San Antonio, TX: The Psychological Corporation, 1993.

38. MISSIUMA, C.; POLATAJKO, H. J. Developmental dyspraxia by any other name: are they all just clumsy children? Am. J. Occup. Ther., v. 49, p. 619-27, 1995.

39. MUTTI, M.; STERLING, H. M.; SPALDING, N. V. Quick neurological screening test. Novato, CA: Academic Therapy Publications, 1978.

40. ROACH, E.G; KEPARTH, N. The purdue perceptualmotor survey. Columbus: Charles E. Merrill Publishing 1966.

41. ROSA NETO, F. Manual de avaliação motora. Porto Alegre: Artes Médicas, 2002.

42. SELLERS, J. S. Clumsiness: review of causes, treatment, and outlook. Occup. Ther. J. Res., v. 15, n. 4, p. 39-55, 1995.

43. STEPHENSON, E.; MCKAY, C.; CHESSON, R. The identification and treatment of motor learning difficulties: parents perception and the role of the therapist. Child Care Health Dev., v. 17, p. 91-113, 1991.

44. WORLD HEALTH ORGANIZATION: International classification of function. Genebra: WHO, 2001.

45. WRIGHT, D. D.; STONE, M. H. Best test design. Chicago: Mesa Press, 1979. 